\title{
EtBr-induced selective degradation of mitochondria occurs via autophagy
}

\author{
YAN LUO, YIDE HU, MINGHUI ZHANG, YU XIAO, ZONGCHANG SONG and YU XU \\ Department of Oncology, The Second Affiliated Hospital, Third Military Medical University, \\ Chongqing 400037, P.R. China
}

Received April 19, 2013; Accepted May 22, 2013

DOI: $10.3892 /$ or.2013.2590

\begin{abstract}
Recent studies have implicated autophagy in numerous cellular responses to stress. During the establishment of human lung cancer cell lines without mitochondrial DNA, a significant depopulation of mitochondria occurred that was accompanied by the loss of the mitochondrial membrane potential. Notably, we observed autophagy in ethidium bromide ( $\mathrm{EtBr}$ )-induced mitochondrial degradation. In the present study, we confirmed the involvement of autophagy in mitochondrial degradation after exposure to a low concentration of EtBr. Lung cancer cells undergoing mitochondrial autophagy exhibited a slower growth rate in vitro and in vivo. Furthermore, the degradation was mediated by the class III phosphatidylinositol 3-kinase (PI3K)-Beclin-1 complex. These findings indicate that autophagy is responsible for EtBr-induced mitochondrial degradation via the PI3K-Beclin-1 signaling pathway.
\end{abstract}

\section{Introduction}

Autophagy is primarily a protective process for the cell (1). Basal levels of autophagy play a critical role in maintaining normal cellular homeostasis by recycling intracellular components $(1,2)$. Currently, the role of autophagy has been extended to human disease and physiology (3). Autophagy was first associated with cancer through the identification and characterization of the Beclin-1 gene, which is thought to be a tumor suppressor (4). Subsequently, several autophagy genes have been determined to play a role in tumorigenesis $(3,5)$.

Correspondence to: Professor Yide Hu, Department of Oncology, The Second Affiliated Hospital, Third Military Medical University, Chongqing 400037, P.R. China

E-mail: huyide_mit@yahoo.com.cn

Abbreviations: LTR, LysoTracker Red; MTG, MitoTracker Green; TMRM, tetramethylrhodamine methyl ester; TEM, transmission electron microscopy; LC3, microtubule-associated protein light chain 3; mtDNA, mitochondrial DNA; EtBr, ethidium bromide; PI3K, phosphatidylinositol 3-kinase

Key words: autophagy, Beclin-1, mitochondria, ethidium bromide, lung cancer cells
Atg proteins are essential for autophagy (6). The C-terminus of newly synthesized microtubule-associated protein light chain 3 (LC3), a well-known Atg protein, is cleaved to generate LC3-I. Subsequently, 22 amino acids from the C-terminus are removed to produce LC3-II, which is recruited to form autophagosomes and serves as a marker of autophagy (7).

Mitochondria are essential and delicate organelles in eukaryotic cells. They function as chemical factories for key metabolic reactions and energy generation and as a communication site for diverse signaling pathways (8). Mitochondrial damage may result in dysfunctional mitochondrial proteins and mitochondrial DNA (mtDNA), sometimes leading to cell death by promoting the intrinsic apoptotic pathways (9). Therefore, accurate control of mitochondrial quality and quantity is necessary for energy metabolism homeostasis and other essential cellular processes. Multiple lines of evidence indicate that the selective degradation of mitochondria by autophagy controls mitochondrial number and health $(10,11)$. Mitochondrial autophagy (termed mitophagy) plays a vital role in selectively degrading superfluous or severely damaged mitochondria $(12,13)$.

Since mitochondria have their own genome, mitochondrial gene knockout cells are utilized to investigate interactions between nuclear and mitochondrial genomes in mitochondrial disease (14). During the establishment of human lung cancer cell lines lacking mtDNA, a progressive depopulation of mitochondria was observed. In the process, autophagy was determined to be over-activated. In the present study, we demonstrated that ethidium bromide $(\mathrm{EtBr})$-induced selective degradation of mitochondria occurred via autophagy. This process was regulated by the phosphatidylinositol 3-kinase (PI3K)-Beclin-1 signaling pathway. EtBr-induced mitochondrial autophagy reduced lung cancer cell growth in vitro and in vivo.

\section{Materials and methods}

Cell culture. The A549, SPC-A1 and H322 human lung cancer cell lines were obtained from the American Type Culture Collection (ATCC). Cells were cultured in ATCC-recommended medium supplemented with 10\% FBS and $100 \mathrm{ng} / \mathrm{ml}$ penicillin and streptomycin. The medium was replaced every other day. For nutrient deprivation, cells were incubated in medium without serum or glucose for $18 \mathrm{~h}$. 
For EtBr treatment, cells were exposed to $250 \mathrm{ng} / \mathrm{ml} \mathrm{EtBr}$ (Biosharp, Korea) for 7 days. The medium was supplemented with $50 \mu \mathrm{g} / \mathrm{ml}$ uridine and $100 \mu \mathrm{g} / \mathrm{ml}$ pyruvate.

Laser scanning confocal microscopy. Cells were loaded with $200 \mathrm{nM}$ tetramethylrhodamine methyl ester (TMRM, T668) or 200 nM LysoTracker Red (LTR, L7528; both from Invitrogen) for $20 \mathrm{~min}$. In other experiments, cells were co-loaded with $200 \mathrm{nM}$ MitoTracker Green (MTG, M7514; Invitrogen) and $200 \mathrm{nM}$ red-fluorescing LTR for $20 \mathrm{~min}$. After fluorescence loading, cells were washed thrice with fresh phosphatebuffered saline (PBS). Confocal images were captured at $2 \mu \mathrm{m}$ intervals using a Leica laser scanning confocal microscope with a Plan Apochromat oil immersion objective lens. The images were merged using LAS AF Lite software (Leica, Germany). Image analysis was performed with Image-Pro Plus5.1 software (IPP, USA).

Transmission electron microscopy (TEM). Cells were fixed in ice-cold electron microscopy-grade glutaraldehyde, rinsed with PBS, post-fixed with $1 \% \mathrm{OsO}_{4}$ in $0.1 \%$ potassium ferricyanide, dehydrated in a graded series of ethanol and embedded in Epon. Ultrathin sections were cut with a diamond knife, stained with $2 \%$ uranyl acetate and Reynold's lead citrate and examined using a Philips EM420 transmission electron microscope.

GFP-LC3 transfection. Cells were transiently transfected with pEGFP-C1-LC3 (Yingrun Biotechnology, China) using Lipofectamine 2000 (Invitrogen). After $24 \mathrm{~h}$, cells were cultured in nutrient-deprived medium or treated with $\mathrm{EtBr}$. Cells were fixed in $4 \%$ formaldehyde for $20 \mathrm{~min}$, washed with PBS, stained with DAPI and observed under a Leica laser scanning confocal microscope.

Western blot analysis. Cells were lysed with SDS lysis buffer (Beyotime, China) containing a protease inhibitor. The protein concentration was measured by the BCA method (Beyotime). An equivalent amount of each denatured protein sample was separated by $12 \%$ SDS-PAGE and electrophoretically transferred onto polyvinylidene difluoride membranes. After blocking with $5 \%$ non-fat milk for $1 \mathrm{~h}$ at room temperature, LC3B (ab48394; Abcam) and Beclin-1 (Epitomics, USA) antibodies were incubated with the membranes at $4^{\circ} \mathrm{C}$ overnight. The membranes were subsequently washed for $30 \mathrm{~min}$ in TBS-Tween 20, incubated with an HRP-conjugated secondary antibody (Beyotime) for $1 \mathrm{~h}$, and observed using chemiluminescence (Beyotime).

Cell proliferation, clonogenic and migration assays. Cells treated with $\mathrm{EtBr}$ for 1, 3, 5 or 7 days were trypsinized and counted. For cell proliferation assays, cells were seeded in 96-well plates $\left(0.5 \times 10^{4} /\right.$ well $)$ and incubated overnight. WST-1 (10 $\mu \mathrm{l} /$ well; Beyotime) was added and the cells were incubated at $37^{\circ} \mathrm{C}$ for $1-2 \mathrm{~h}$. Differences in absorbance were measured using a microplate reader. For clonogenic assays, cells were seeded in 6 -well plates $\left(10^{3} /\right.$ well $)$ and then incubated in selective growth medium (supplemented with $50 \mu \mathrm{g} / \mathrm{ml}$ uridine and $100 \mu \mathrm{g} / \mathrm{ml}$ pyruvate) for 10 days. Cells were fixed with methanol for $15 \mathrm{~min}$ and stained with $0.1 \%$ crystal violet for
10 min. Colonies were visualized and counted using light microscopy.

For cell migration assays, cells $\left(1 \times 10^{4} ; 100 \mu \mathrm{l}\right)$ were seeded in the upper chambers of 24-well Transwell plates $(8-\mu \mathrm{m}$ pore size; Corning, NY, USA), and medium supplemented with $12 \%$ FBS $(800 \mu \mathrm{l})$ was added to the bottom chambers. After $20-22 \mathrm{~h}$, non-migrated cells on the upper side of the filter membrane were gently removed. Cells that had migrated to the lower side of the insert membrane were fixed with methanol for $15 \mathrm{~min}$ and stained with $1 \%$ crystal violet for $10 \mathrm{~min}$. The migrated cells were counted in five random fields using a microscope.

In vivo analysis of tumor growth. The animal studies were performed in accordance with the guidelines of the Third Military Medical University Animal Care and Use Committee. NOD/SCID mice (5 mice/group) were injected subcutaneously in the left flank with $1 \times 10^{6} \mathrm{EtBr}$-treated cells suspended in $200 \mu 1$ PBS. Tumor volume was measured with calipers twice a week for 6 weeks, after which the mice were sacrificed. Tumors were removed and photographed.

Statistical analysis. Data are presented as the mean \pm SD and the 95\% confidence interval. Independent t-tests were utilized to evaluate the data. Statistical significance was defined as $\mathrm{P}<0.05$.

\section{Results}

EtBr inhibits lung cancer cell growth in vitro and in vivo without significantly inducing apoptosis. During the process of knocking out mitochondrial genes, the biological behavior of EtBr-treated cells was assessed. In vitro cell proliferation, clonogenic and migration assays demonstrated that EtBr inhibited lung cancer cell growth and migration in a time-dependent manner, but there was no significant increase in apoptotic events in EtBr-treated cells (Fig. 1A). The PI staining results demonstrated that EtBr-treated cells underwent cell cycle arrest (Fig. 1B). In vivo, EtBr-treated cells grew more slowly than untreated control cells in the lung cancer xenograft models (Fig. 1C).

Increased LTR uptake and reduced TMRM fluorescence in EtBr-treated cells. At different time points after exposure to $250 \mathrm{ng} / \mathrm{ml} \mathrm{EtBr}$, A549, SPC-A1 and H322 cells were loaded with Lyso-Tracker Red (LTR) to detect acidic organelles. Compared with the control cells, the number of LTR-labeled organelles in EtBr-treated cells significantly increased from $13 \pm 2.7$ to $78 \pm 4.2$ per optical section by day 1 but decreased to $52 \pm 4.3$ by day 3 ( $\mathrm{n}=5$ optical sections; $\mathrm{P}<0.01$ ) (Fig. $2 \mathrm{~A}$ ). The same changes were observed in the nutrient-deprived cells $(\mathrm{n}=5$ optical sections; $\mathrm{P}<0.01)$ (Fig. $2 \mathrm{~B}$ ). Subsequently, cells were loaded with TMRM to detect polarized mitochondria. The results indicated that the mitochondrial membrane potential (MMP) decreased in a time-dependent manner ( $\mathrm{n}=5$ optical sections; $\mathrm{P}<0.05$ ) (Fig. $2 \mathrm{C}$ and $\mathrm{D}$ ). The decreased MMP was confirmed by flow cytometry using JC-1 staining (Fig. 2E-G). Alterations in mitochondrial size and shape were identified by TMRM microscopy. The mitochondria, typically homogeneous in size, became large and branched after treatment with EtBr. 
A
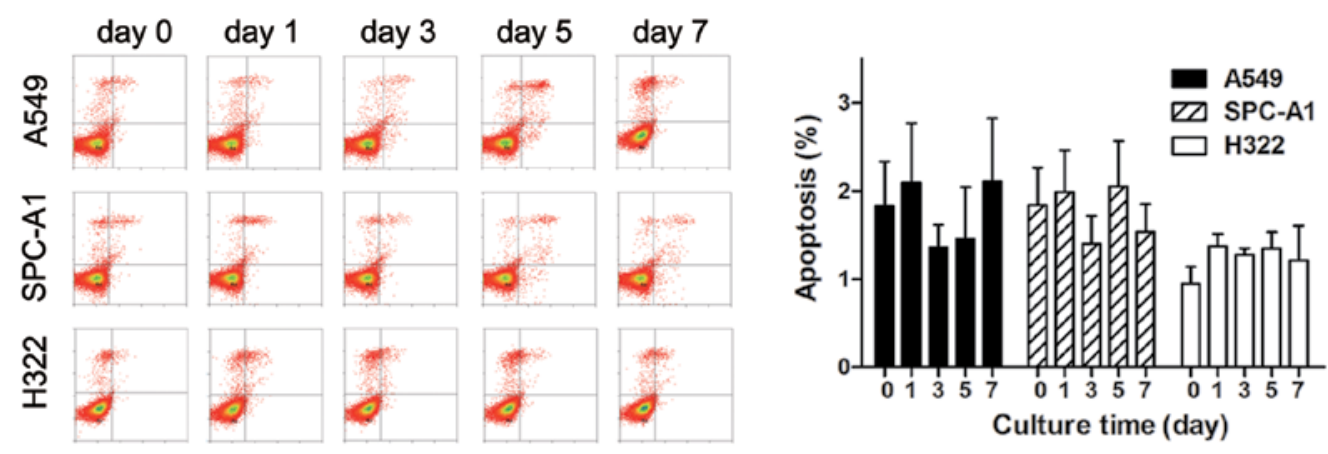

B
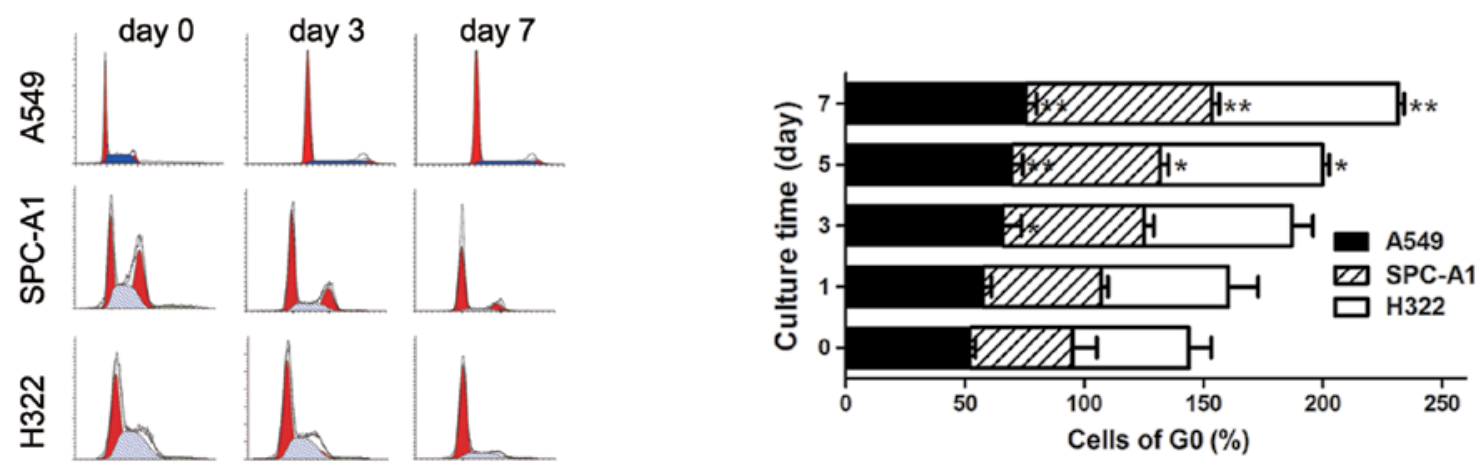

C

day 0

day 1

day 3

day 5

day 7
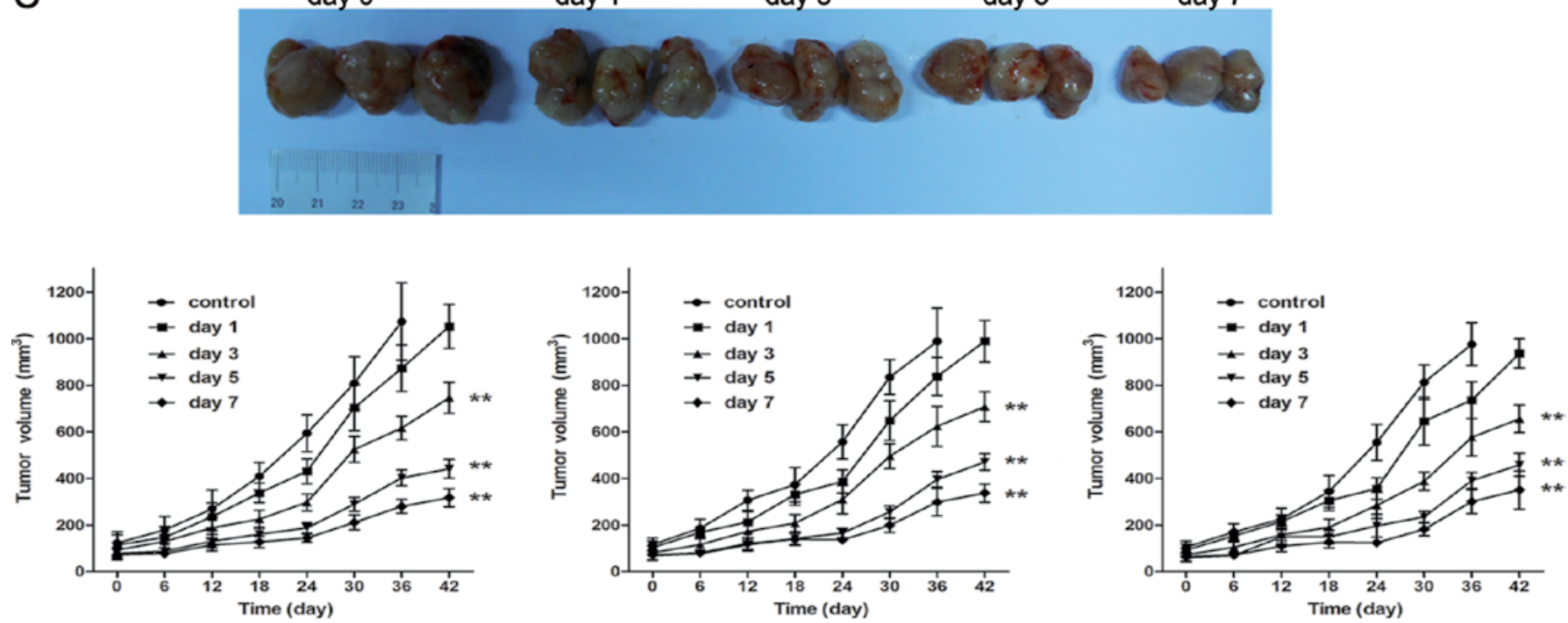

Figure 1. Effects of ethidium bromide (EtBr) on cell growth and apoptosis in lung cancer cells. (A) Flow cytometric analysis was performed to detect early apoptotic events in cells treated with EtBr for 1,3,5 or 7 days. (B) Cell cycle distribution of EtBr-treated cells was assessed by flow cytometry using PI staining. Numbers indicate the percentage of cells in the G0 phase. (C) Approximately $10^{6}$ A549, SPC-A1 and H322 cells treated with EtBr for 1, 3, 5 or 7 days were injected into the left flank of the mice. Tumor sizes were measured twice a week for 6 weeks, and tumor volumes were calculated. Data are presented as the mean $\pm \mathrm{SD}\left({ }^{*} \mathrm{P}<0.05 ;{ }^{* * *} \mathrm{P}<0.01\right)$.

Since a significant depopulation of polarized mitochondria was observed in EtBr-treated cells, the mtDNA content and the mRNA expression of cytochrome $c$ oxidase subunit II (COX II) were measured by quantitative real-time PCR. The results demonstrated that these two markers significantly decreased in a time-dependent manner after EtBr treatment (data not shown).

Mitochondrial degradation by LTR-labeled organelles in cells treated with EtBr. To observe the movement of mitochondria into acidic lysosomal structures in EtBr-treated cells, cells were co-loaded with red-fluorescing LTR and green-fluorescing MTG. As illustrated in Fig. 3, there was little co-localization of LTR- and MTG-positive structures in the control cells. After 1 day of treatment with $\mathrm{EtBr}$, the number of dual-labeled structures per section increased from $3.3 \pm 1.5,4.7 \pm 0.6$ and $5.7 \pm 1.5$ to $31 \pm 4.6,51 \pm 5.6$ and $46.3 \pm 6.5$ in the A549, SPC-A1 and $\mathrm{H} 322$ cells, respectively $(\mathrm{n}=5$ sections; $\mathrm{P}<0.01)$ (Fig. $3 \mathrm{~A})$. In cells starved for $18 \mathrm{~h}$, a significant increase in co-localization was observed ( $\mathrm{n}=5$ sections; $\mathrm{P}<0.01)$ (Fig. $3 \mathrm{~A}$ ). Compared with starvation, EtBr treatment more dramatically increased the co-localization of MTG- and LTR-positive structures (Fig. 3B). 
A

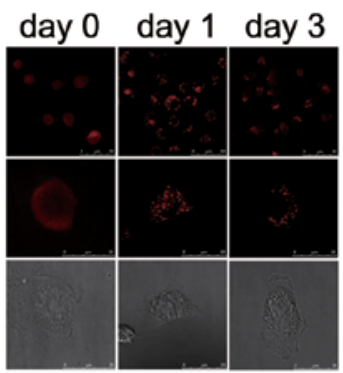

$\mathrm{EtBr}$

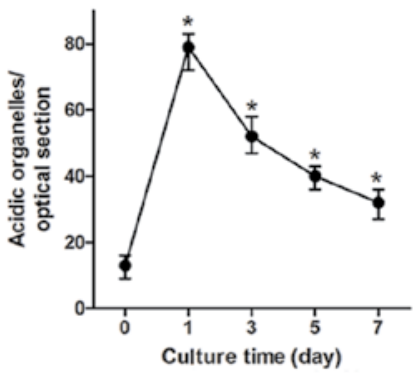

B
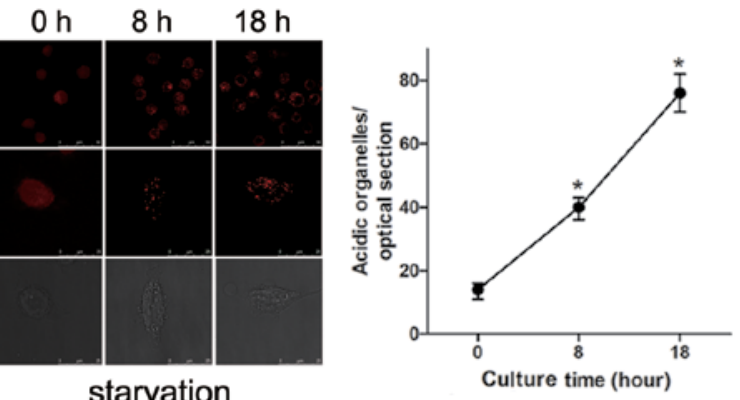

C
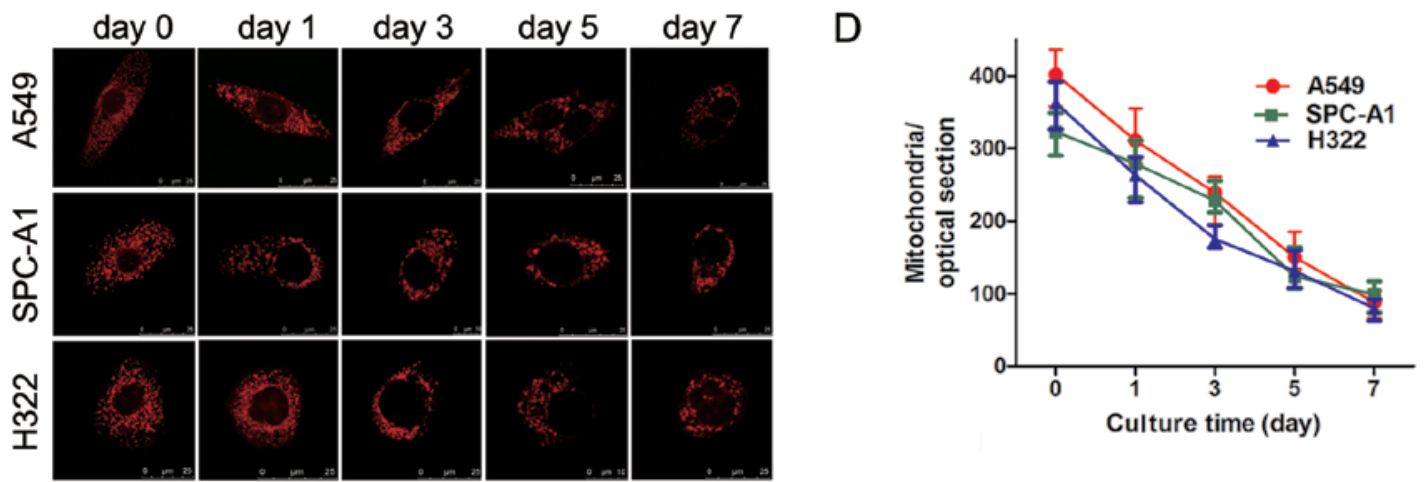

E

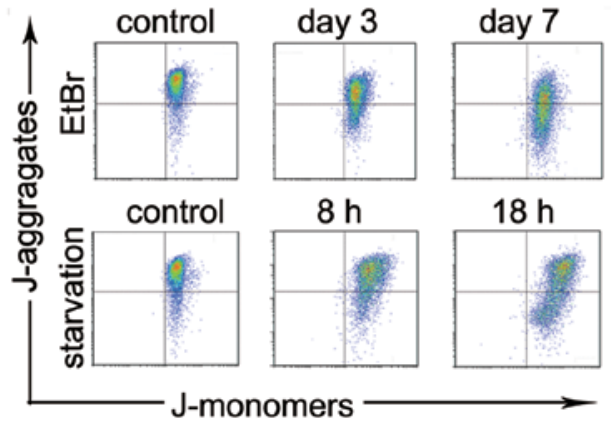

$\mathrm{F}$

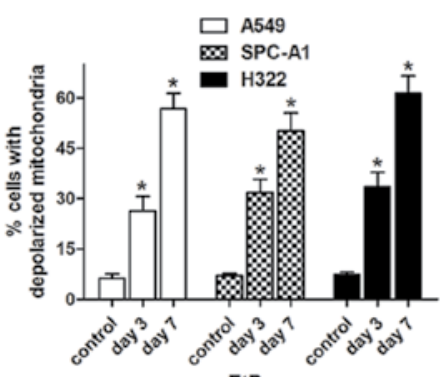

EtBr
G

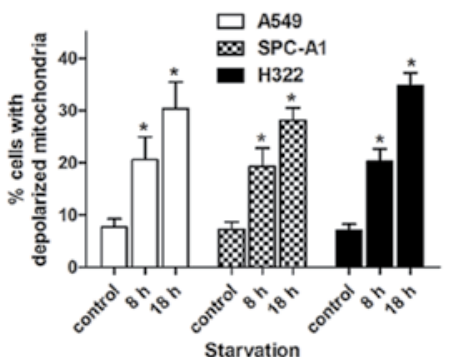

Figure 2. Increased acidic organelles and decreased mitochondrial content during ethidium bromide (EtBr) treatment. (A) Cells were incubated with $200 \mathrm{nM}$ LysoTracker Red (LTR) for $20 \mathrm{~min}$; subsequently, confocal fluorescence and corresponding bright field images were captured. The average number of LTR-labeled organelles per optical section is plotted. (B) Cells starved for 8 or $18 \mathrm{~h}$ were stained with $200 \mathrm{nM} \mathrm{LTR}$ for 20 min. The images were collected and analyzed. (C) Cells treated with EtBr for 1,3,5 or 7 days were loaded with tetramethylrhodamine methyl ester (TMRM). Representative images are presented (D) TMRM-labeled mitochondria were quantified at different time points, and the data are plotted. (E-G) Mitochondrial membrane potential was determined by biparametric flow cytometry after staining with JC-1. Data are presented as the mean $\pm \mathrm{SD}\left({ }^{*} \mathrm{P}<0.01\right)$.

A

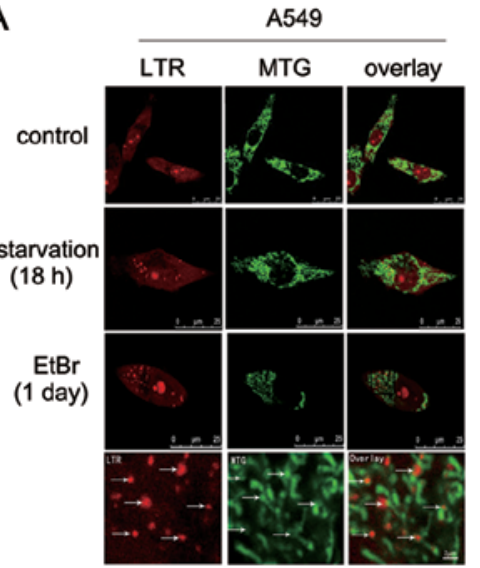

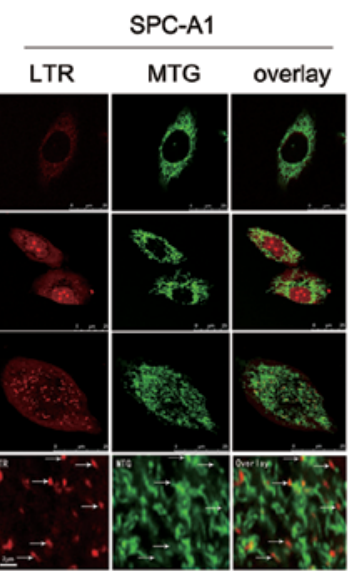

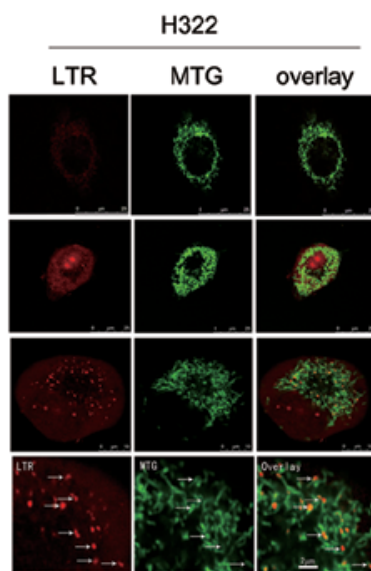

B

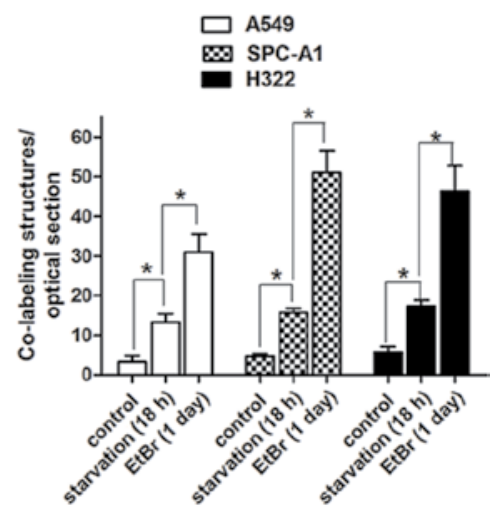

Figure 3. Mitochondria entrapped in acidic organelles after starvation and ethidium bromide (EtBr) treatment. (A) A549, SPC-A1 and H322 cells starved for 8 or $18 \mathrm{~h}$ or treated with EtBr for 1, 3, 5 or 7 days were co-labeled with LysoTracker Red (LTR) and MitoTracker Green (MTG) and imaged. Time points were selected to represent the process of mitochondrial degradation by acidic organelles. The bottom panels show overlaid images at a higher power. The arrows indicate red-LTR, green-MTG and an orange-yellow superimposition of LTR and MTG. (B) Co-localized structures were quantified and analyzed. The data are presented as the mean $\pm \mathrm{SD}\left({ }^{*} \mathrm{P}<0.01\right)$. 


\section{A}

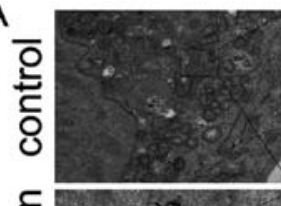

$\frac{\text { 음 }}{\frac{\pi}{\pi}}$

๓⿳亠口冋乚

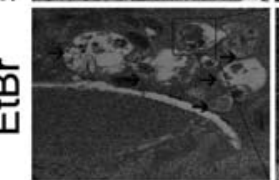

C

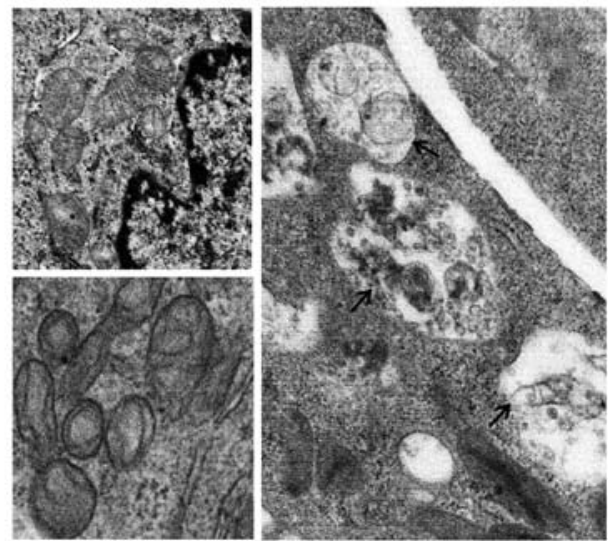

B
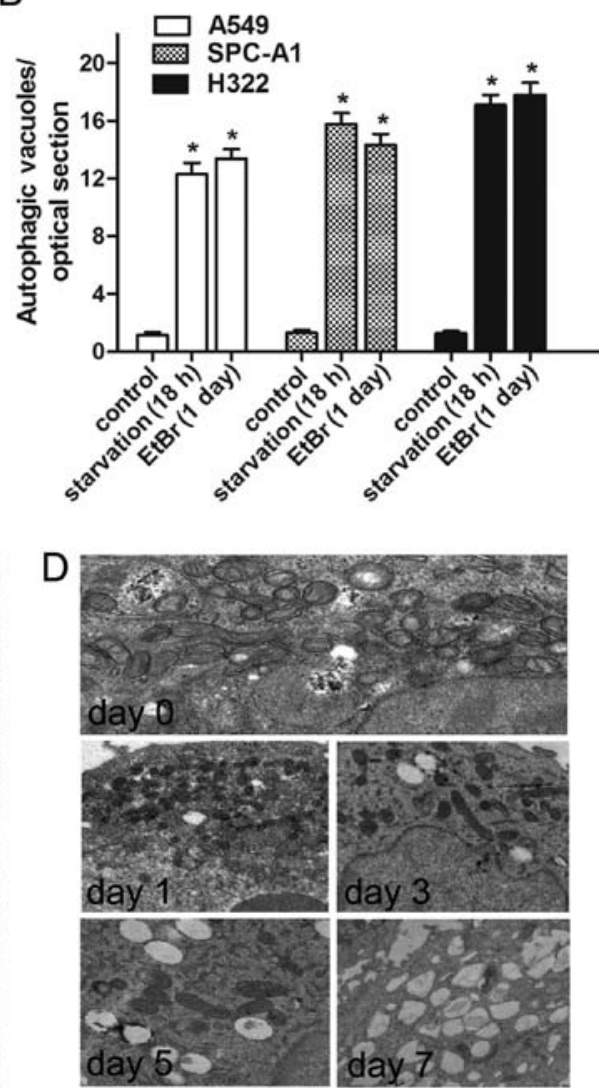

Figure 4. Electron microscopy of cells that were starved and treated with ethidium bromide (EtBr). (A) Representative electron microscopic images of autophagic vacuoles with cytoplasmic cargo (black arrows) are presented. (B) The number of autophagic vacuoles per optical section was analyzed ( $\mathrm{n}=30$ sections). (C) Compared with the control cells, EtBr-treated cells exhibited an aberrant mitochondrial appearance with partial cristae. The arrows indicate autophagic vacuoles entrapping mitochondria. (D) The number of mitochondria and cytoplasmic lipid droplets in cells treated with EtBr for $1,3,5$ or 7 days is shown. The data are presented as the mean $\pm \mathrm{SD}\left({ }^{*} \mathrm{P}<0.01\right)$.

Transmission electron microscopy to identify autophagic ultrastructures. To confirm the mitochondrial degradation via autophagy, TEM was performed. In cells starved for $18 \mathrm{~h}$, the presence of autophagic vacuoles with cytoplasmic content (Fig. 4A, middle, arrows) increased significantly when compared with the control cells $(\mathrm{n}=30$ cells; $\mathrm{P}<0.01)$ (Fig. 4A and B). Similarly, more mitochondria encapsulated in double-membrane vesicles (Fig. 4A, bottom, arrows) were observed after EtBr treatment. Consistent with the TMRM fluorescence microscopy results, there was an obvious depopulation of mitochondria from day 1 to day 7. Morphological alterations in the internal structures of the remaining mitochondria were identified. After 7 days of EtBr treatment, the mitochondria exhibited aberrant phenotypes with partial or complete loss of regular crista patterns. In contrast, the untreated control cells possessed typical elongated mitochondria with parallel cristae (Fig. 4C). In addition, a significant increase in cytoplasmic lipid droplets was observed in $\mathrm{EtBr}$ treated cells (Fig. 4D).

Recruitment of LC3-II to autophagosomes during mitochondrial degradation via autophagy. To monitor autophagic activity, the conversion of endogenous LC3-I to LC3-II was assessed. An expression vector encoding pEGFP-C1-LC3 was utilized. In cells treated for $3 \mathrm{~h}$ with $\mathrm{EtBr}$, the expression level of GFP-LC3 began to increase. By day 1, the number of GFP-LC3 punctae reached a maximum. After $24 \mathrm{~h}$, the number of green LC3 dots decreased but remained significantly higher than that in the control cells (Fig. 5A and B). Similar patterns of LC3-II conversion were identified by western blot analysis (Fig. 5F). We examined whether 3-MA, an autophagy inhibitor, inhibits EtBr-induced autophagy. The results indicated that EtBr-induced autophagy was inhibited by 3 -MA $(\mathrm{P}<0.05)$ (Fig. 5D and $\mathrm{G})$. In cells starved for 8 or $18 \mathrm{~h}$, the number of GFP-LC3 dots increased compared with the control cells (Fig. 5A and C). A significant conversion of LC3-I to LC3-II was detected by western blotting (Fig. 5E), and starvation-induced LC3-II conversion was time-dependent. As expected, 3-MA markedly decreased LC3-II recruitment in the starved cells $(\mathrm{P}<0.05)$ (Fig. 5D).

The expression level of Beclin-1 was analyzed as it has been reported to be required for the initial formation of autophagosomes during autophagy (15). During culture in nutrient deprivation medium, cancer cells exhibited increased Beclin-1 protein (Fig. 5H). A similar increase in Beclin-1 protein was observed in cells treated with EtBr (Fig. 5I); this increase was inhibited by 3-MA, an autophagy inhibitor (Fig. 5J).

\section{Discussion}

Unlike nuclear DNA, mtDNA lacks protection by histones, and mitochondria have a limited capacity for self-repair, 
A

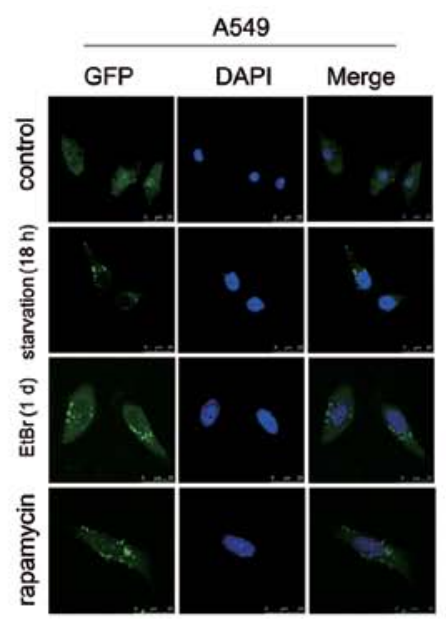

B

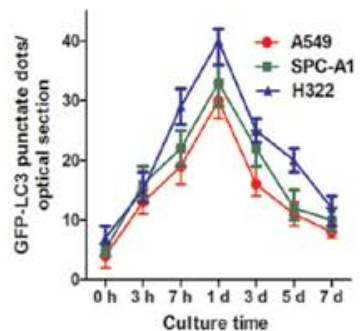

$E$

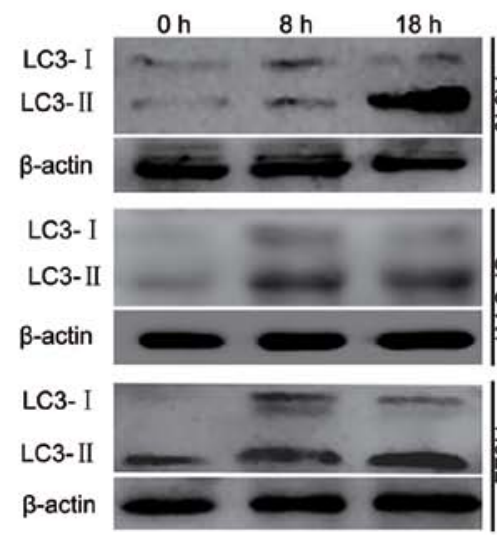

$\mathrm{H}$

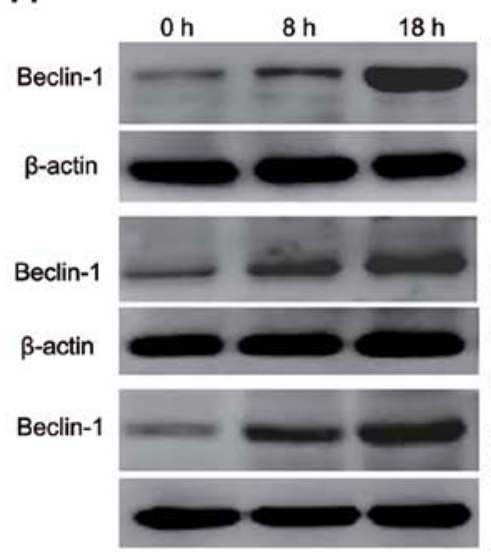

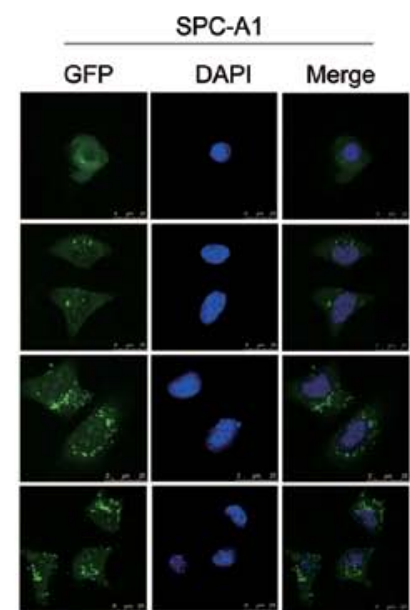

C

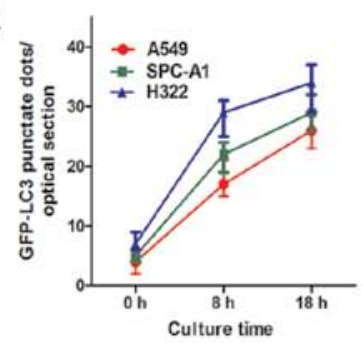

F
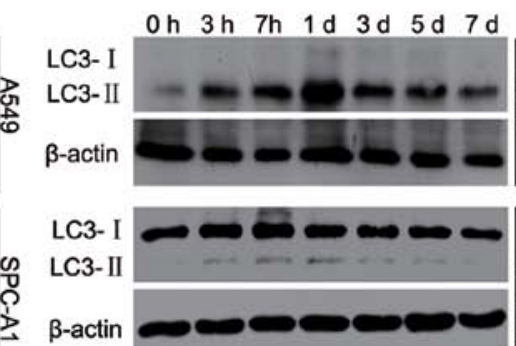

LC3- I

LC3-II

$\beta$-actin

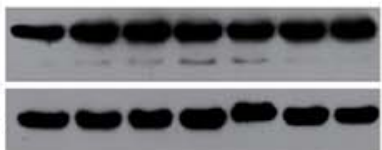

I
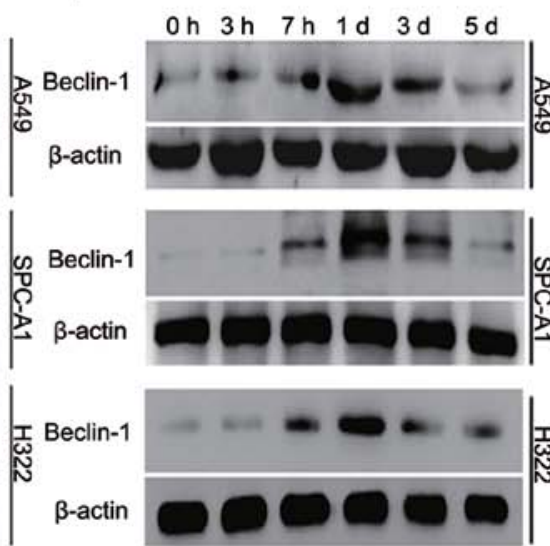

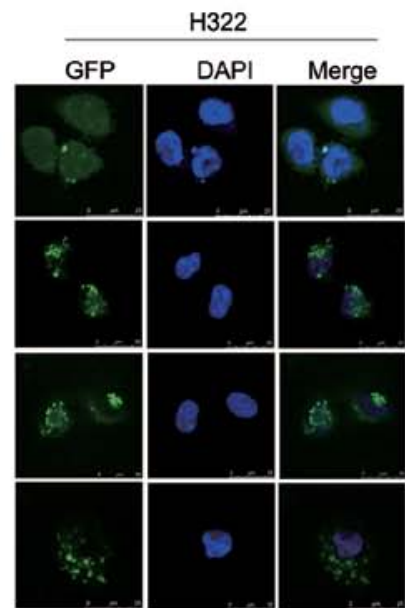

D

$\square$ control $\square \mathrm{EtBr}+3-\mathrm{MA}$

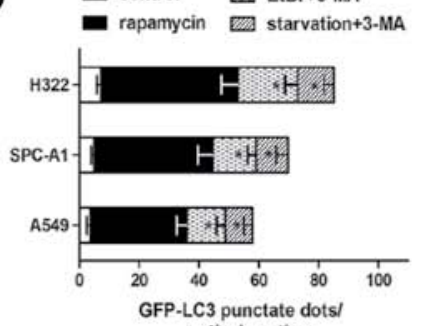

optical section

$\begin{array}{llll}\mathrm{EtBr}- & + & - & + \\ \text { r-MA }- & + & + & -\end{array}$

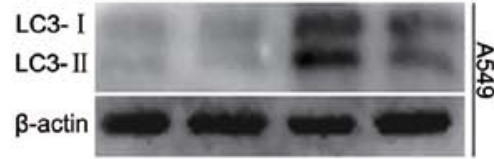

LC3-

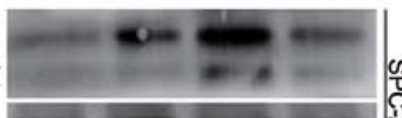

$\beta$-actin

LC3- I

LC3-II

$\beta$-actin

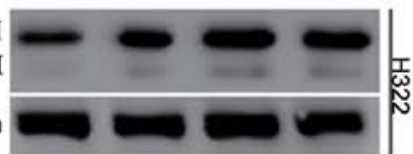

J EtBr - + - + rapamycin : $\div+\div$
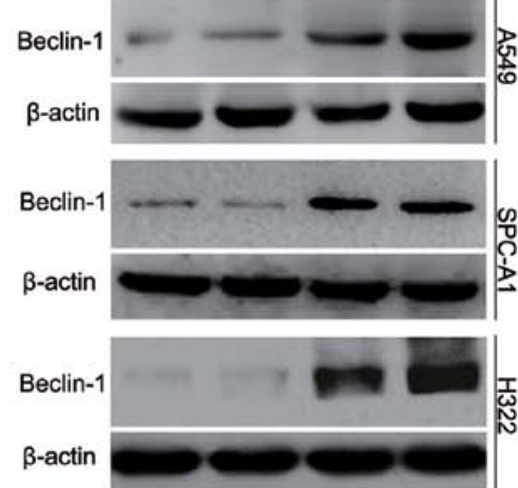

Figure 5. Increased GFP-microtubule-associated protein light chain 3 (LC3) punctae and LC3-II conversion in ethidium bromide (EtBr)-treated cells. (A) Cells were transfected with pEGFP-C1-LC3, treated with EtBr for 1 day or cultured in nutrient-deprived medium for $18 \mathrm{~h}$ and imaged with a fluorescence microscope. (B and C) GFP-LC3 punctae were quantified and expressed as the number of green fluorescent dots per optical section (n=5 sections). (D) GFP-LC3 punctae were quantified in cells pre-treated with 3-MA. (E) Starvation-induced autophagy in lung cancer cells was revealed by western blot analysis of LC3-II conversion. Cells were cultured in nutrient-deprived medium for 8 or $18 \mathrm{~h}$. (F and G) EtBr treatment led to LC3-II conversion as demonstrated by western blotting. Cells were treated with EtBr for 3, $7 \mathrm{~h}$ and 1,3,5 or 7 days. (H) Western blot analysis of Beclin-1 in cells starved for 8 or $18 \mathrm{~h}$ is illustrated. (I and $\mathrm{J}$ ) The protein level of Beclin-1 was examined at various time points after EtBr treatment. Rapamycin $(0.5 \mu \mathrm{M})$ and 3-MA $(10 \mathrm{mM})$ were used to induce and inhibit autophagy, respectively. The data are presented as the mean $\pm \mathrm{SD}($ " $\mathrm{P}<0.05$, compared with cells without 3-MA pre-treatment). 
making mtDNA more susceptible to damage (16). Typically, a low concentration of EtBr has been used to inhibit mtDNA replication and transcription (17-19). During the establishment of lung cancer cell lines without mtDNA, a progressive depopulation of mitochondria was observed that was accompanied by increased LTR uptake and co-localization of LTR- and MTG-positive structures. Moreover, autophagosomal structures containing mitochondrial remnants were directly observed with an electron microscope. The expression of LC3-II and Beclin-1 significantly increased, but these increases were inhibited by 3-MA, an autophagy inhibitor. Our findings suggest that autophagy was responsible for mitochondrial degradation after EtBr exposure, most likely through the PI3K-Beclin-1 pathway.

TMRM is a cell-permeant, cationic, red-orange fluorophore that localizes to mitochondria in response to a high negative membrane potential (20). In the present study, decreased TMRM red fluorescence was observed by confocal microscopy, suggesting a significant loss of MMP. Electron microscopy provided solid evidence of mitochondrial depopulation. Moreover, the mtDNA copies and mRNA levels of COX were significantly decreased in EtBr-treated cells. Taken together, $\mathrm{EtBr}$ treatment led to mitochondrial degradation. Moreover, alterations in mitochondrial ultrastructure were identified. In EtBr-treated cells, the remaining mitochondria became large and branched compared with the spherical, oval or short rod-like shape of the control cells. The cristae inside the mitochondria were less abundant and shorter.

During the loss of mitochondria, we observed a significant increase in acidic organelles. This was verified by an increased uptake of LTR, an acidotrophic fluorescent probe (21). LTR labels all acidic organelles, including lysosomes and late endosomes, not just autophagosomes. However,Rodriguez-Enriquezetal (21)and Kim and Lemasters (22) reported that, in the context of nutrient deprivation-induced autophagy, the increased number of LTR fluorescent dots represented an increase in autophagosomes and autolysosomes. Therefore, we believe that the increased LTR uptake in EtBr-treated cells was due to an increase in autophagosomes and autolysosomes. The movement of MTG-labeled mitochondria into LTR-labeled acidic autolysosomal structures was visualized by confocal microscopy. The autophagosomal structures containing mitochondrial remnants were observed in the cytoplasm by electron microscopy. These findings suggest that mitochondrial degradation in cells treated with EtBr was accomplished via an autophagic pathway. Well-established nutrient deprivation-induced autophagy was employed as a control. As previously reported, mitochondria occupy $\sim 5-6 \%$ of the cytoplasmic volume $(23,24)$, thus mitochondria were a major target for autophagic digestion after nutrient deprivation.

LC3-II conversion is usually studied as an indicator of autophagic activity. LC3-tagged GFP has been utilized to monitor autophagy through direct fluorescence microscopy (25). In complete growth medium, lung cancer cells transfected with GFP-LC3 exhibited a diffuse distribution of green fluorescence in the cytosol. In cells treated with $\mathrm{EtBr}$, a marked increase in GFP-LC3 punctae was observed. This characteristic LC3-II conversion was verified by western blot analysis. Similar changes in this autophagic marker were identified in starved cells. Furthermore, GFP-LC3 patches containing TMRM-labeled polarized mitochondria were observed in GFP-LC3 transgenic hepatocytes (22). This convinced us that selective mitochondrial degradation via autophagy was involved in nutrient deprivation-induced autophagy. It has been reported that 3-MA can inhibit class III PI3K and subsequently suppress LC3-II conversion under starvation conditions $(26,27)$, which is important in clarifying the effects of autophagy. Our data demonstrated that 3-MA inhibited the formation of GFP-LC3 punctae as well as the accumulation of LC3-II protein in EtBr-treated cells. Therefore, since increased Beclin-1 expression following EtBr treatment could be inhibited by 3-MA, EtBr-induced mitochondrial autophagy was activated through the class III PI3K pathway.

Currently, the role of autophagy in keeping cells alive or inducing cell death is controversial (28-30). We believe that excessive autophagy breaks the delicate balance between cell survival and cell death. In our study, mitochondrial autophagy was over-activated in lung cancer cells by continuous exposure to $\mathrm{EtBr}$, which resulted in a slower growth rate in vitro and in vivo. As Kulawiec et al (31), Yu et al (32) and Singh (33) reported, mitochondrial damage can result in cell cycle arrest and prevent the initiation of apoptosis. In the present study, EtBr-induced mitochondrial damage may activate the mito-checkpoint to maintain the mitochondrial integrity of lung cancer cells. mtDNA dysfunction was reported to cause chromosomal instability in the nucleus $(34,35)$. Therefore, interference with the normal function of nDNA was thought to explain the decreased tumorigenicity of EtBr-treated lung cancer cells. In the present study, our data demonstrated that excessive mitochondrial degradation via autophagy was, at least in part, responsible for inhibiting the cell growth and proliferation of EtBr-treated lung cancer cells.

In conclusion, we demonstrated that autophagy is responsible for mitochondrial degradation in lung cancer cell lines exposed to a low concentration of EtBr. We also demonstrated that the class III PI3K-Beclin-1 complex is involved in mediating EtBr-induced mitochondrial autophagy. The present data indicate that mitochondrial autophagy can inhibit cell proliferation, migration, and tumorigenesis but cannot induce significant apoptosis or cell death. Our findings provide new insight into the effects of mitochondrial autophagy on lung cancer cells.

\section{Acknowledgements}

We would like to thank Jin Peng and Jie He (Central Laboratory, The Second Affiliated Hospital of Third Military Medical University, Chongqing, China) for their tremendous help with laser confocal scanning microscopy and Halei Sheng for her technical assistance with flow cytometry (Central Laboratory, The Second Affiliated Hospital of Third Military Medical University, Chongqing, China).

\section{References}

1. Mizushima N, Levine B, Cuervo AM and Klionsky DJ: Autophagy fights disease through cellular self-digestion. Nature 451: 1069-1075, 2008.

2. Eskelinen EL and Saftig P: Autophagy: a lysosomal degradation pathway with a central role in health and disease. Biochim Biophys Acta 1793: 664-673, 2009. 
3. Huang $\mathrm{J}$ and Klionsky DJ: Autophagy and human disease. Cell Cycle 6: 1837-1849, 2007.

4. Yue Z, Jin S, Yang C, Levine AJ and Heintz N: Beclin 1, an autophagy gene essential for early embryonic development, is a haploinsufficient tumor suppressor. Proc Natl Acad Sci USA 100: 15077-15082, 2003.

5. Jin S: Autophagy, mitochondrial quality control, and oncogenesis. Autophagy 2: 80-84, 2006.

6. Mizushima N, Yoshimori T and Ohsumi Y: The role of Atg proteins in autophagosome formation. Annu Rev Cell Dev Biol 27: 107-132, 2011.

7. Kabeya Y, Mizushima N, Ueno T, Yamamoto A, Kirisako T, Noda T, Kominami E, Ohsumi Y and Yoshimori T: LC3, a mammalian homologue of yeast Apg8p, is localized in autophagosome membrane after processing. EMBO J 19: 5720-5728, 2000.

8. Malhotra JD and Kaufman RJ: ER stress and its functional link to mitochondria: role in cell survival and death. Cold Spring Harb Perspect Biol 3: a004424, 2011.

9. Gupta KJ, Igamberdiev AU and Mur LA: NO and ROS homeostasis in mitochondria: a central role for alternative oxidase. New Phytol 195: 1-3, 2012.

10. Lee J, Giordano S and Zhang J: Autophagy, mitochondria and oxidative stress: cross-talk and redox signalling. Biochem J 441: 523-540, 2012

11. Rambold AS and Lippincott-Schwartz J: Mechanisms of mitochondria and autophagy crosstalk. Cell Cycle 10: 4032-4038, 2011.

12. Bhatia-Kiššová I and Camougrand N: Mitophagy: a process that adapts to the cell physiology. Int J Biochem Cell Biol 45: 30-33, 2013.

13. Ashrafi G and Schwarz TL: The pathways of mitophagy for quality control and clearance of mitochondria. Cell Death Differ 20: 31-42, 2013 .

14. Hashiguchi K and Zhang-Akiyama QM: Establishment of human cell lines lacking mitochondrial DNA. Methods Mol Biol 554: 383-391, 2009.

15. Kang R, Zeh HJ, Lotze MT and Tang D: The Beclin 1 network regulates autophagy and apoptosis. Cell Death Differ 18: 571-580, 2011.

16. Alexeyev MF: Is there more to aging than mitochondrial DNA and reactive oxygen species? FEBS J 276: 5768-5787, 2009.

17. Garcia N,Hernández-Esquivel L,Zazueta C,Martínez-Abundis E, Pavón $\mathrm{N}$ and Chávez E: Induction of mitochondrial permeability transition by the DNA-intercalating cationic dye ethidium bromide. J Biochem 146: 887-894, 2009.

18. Armand R, Channon JY, Kintner J, White KA, Miselis KA, Perez RP and Lewis LD: The effects of ethidium bromide induced loss of mitochondrial DNA on mitochondrial phenotype and ultrastructure in a human leukemia T-cell line (MOLT-4 cells). Toxicol Appl Pharmacol 196: 68-79, 2004.

19. von Wurmb-Schwark N, Cavelier L and Cortopassi GA: A low dose of ethidium bromide leads to an increase of total mitochondrial DNA while higher concentrations induce the mtDNA 4997 deletion in a human neuronal cell line. Mutat Res 596: 57-63, 2006.
20. Chazotte B: Labeling mitochondria with TMRM or TMRE. Cold Spring Harb Protoc 2011: 895-897, 2011.

21. Rodriguez-Enriquez S, Kim I, Currin RT and Lemasters JJ: Tracker dyes to probe mitochondrial autophagy (mitophagy) in rat hepatocytes. Autophagy 2: 39-46, 2006.

22. Kim I and Lemasters JJ: Mitochondrial degradation by autophagy (mitophagy) in GFP-LC3 transgenic hepatocytes during nutrient deprivation. Am J Physiol Cell Physiol 300: C308-C317, 2011.

23. Dewey WC and Fuhr MA: Quantification of mitochondria during the cell cycle of Chinese hamster cells. Exp Cell Res 99: 23-30, 1976.

24. Segui-Simarro JM, Coronado MJ and Staehelin LA: The mitochondrial cycle of Arabidopsis shoot apical meristem and leaf primordium meristematic cells is defined by a perinuclear tentaculate/cage-like mitochondrion. Plant Physiol 148: 1380-1393, 2008.

25. Mizushima N, Yoshimori $\mathrm{T}$ and Levine B: Methods in mammalian autophagy research. Cell 140: 313-326, 2010.

26. Wu YT, Tan HL, Shui G, Bauvy C, Huang Q, Wenk MR, Ong CN, Codogno P and Shen HM: Dual role of 3-methyladenine in modulation of autophagy via different temporal patterns of inhibition on class I and III phosphoinositide 3-kinase. J Biol Chem 285: 10850-10861, 2010.

27. Petiot A, Ogier-Denis E, Blommaart EF, Meijer AJ and Codogno P: Distinct classes of phosphatidylinositol 3'-kinases are involved in signaling pathways that control macroautophagy in HT-29 cells. J Biol Chem 275: 992-998, 2000.

28. Chen $\mathrm{N}$ and Karantza-Wadsworth V: Role and regulation of autophagy in cancer. Biochim Biophys Acta 1793: 1516-1523, 2009.

29. White E, Karp C, Strohecker AM, Guo Y and Mathew R: Role of autophagy in suppression of inflammation and cancer. Curr Opin Cell Biol 22: 212-217, 2010

30. Gorski SM, Ries J and Lum JJ: Targeting autophagy: the Achilles' heel of cancer. Autophagy 8: 1279-1280, 2012.

31. Kulawiec M, Ayyasamy V and Singh KK: p53 regulates mtDNA copy number and mitocheckpoint pathway. J Carcinog 8: 8, 2009.

32. Yu M, Shi Y, Wei X, Yang Y, Zhou Y, Hao X, Zhang N and Niu R: Depletion of mitochondrial DNA by ethidium bromide treatment inhibits the proliferation and tumorigenesis of T47D human breast cancer cells. Toxicol Lett 170: 83-93, 2007.

33. Singh KK: Mitochondria damage checkpoint, aging, and cancer. Ann NY Acad Sci 1067: 182-190, 2006.

34. Amuthan G, Biswas G, Ananadatheerthavarada HK, Vijayasarathy C, Shephard HM and Avadhani NG: Mitochondrial stress-induced calcium signaling, phenotypic changes and invasive behavior in human lung carcinoma A549 cells. Oncogene 21: 7839-7849, 2002.

35. Chen D, Xue W and Xiang J: The intra-nucleus integration of mitochondrial DNA (mtDNA) in cervical mucosa cells and its relation with c-myc expression. J Exp Clin Cancer Res 27: 36, 2008. 